Research Article

\title{
Mobile Information System for English Teaching Based on Edge Computing and VR
}

\author{
Yanfang Zhou \\ School of Foreign Languages, Xiangnan University, Chenzhou 423000, Hunan, China \\ Correspondence should be addressed to Yanfang Zhou; xnxyzyf@xnu.edu.cn
}

Received 25 August 2021; Accepted 28 October 2021; Published 23 November 2021

Academic Editor: Sang-Bing Tsai

Copyright (c) 2021 Yanfang Zhou. This is an open access article distributed under the Creative Commons Attribution License, which permits unrestricted use, distribution, and reproduction in any medium, provided the original work is properly cited.

\begin{abstract}
There are many difficulties in using cloud computing technology to solve this problem, and edge computing technology just provides an effective way to solve this problem. Changing from two-dimensional to three-dimensional will make education more realistic. This article uses the VR technology algorithm to design and analyze the English teaching system, combined with the current environment and requirements, in the construction of teaching resources, in order to clarify the direction in practical application. Secondly, according to the scene of human behavior recognition, a human behavior data collection, processing and analysis system based on edge computing is designed and implemented. And on the basis of detailed analysis of VR technology, the theoretical basis of VR technology applied to experiment teaching is discussed, and the development of virtual experiment is analyzed concretely using systematic teaching design ideas. VR technology greatly stimulates students' learning motivation but also significantly improves their interest in learning. In addition, the application of virtual technology has greatly improved students' learning enthusiasm. According to the findings of this article, based on the application of virtual reality technology, students' English comprehension skills have increased by $19 \%$, and the degree of resource sharing has increased by $11 \%$.
\end{abstract}

\section{Introduction}

The application range of virtual reality technology is expanding day by day. Virtual reality has gradually entered the daily activities of education and teaching from pure scientific research, and a new situation of "virtual teaching" has appeared in professional fields such as military applications. These applications are also demonstrations of hightech education and concrete demonstrations of information technology and educational modernization. Practice has proved that VR technology can construct immersive, fantasy, and interactive three-dimensional virtual physical scenes. On this platform, dynamic interactive perception of objects can be realized, and the communication and sharing of virtual scenes can be combined with network technology. Especially the application in virtual reality teaching can alleviate the contradiction between the existing experimental equipment and the needs of students to a certain extent. Three-dimensional modeling technology is used to construct the experimental equipment needed in the experimental teaching process, allowing students to "real" experience the performance of the experimental equipment. Using virtual reality language combined with programming ideas can simulate the experiment process and the phenomenon in the experiment. Students can accurately understand these phenomena and master the laws of things so as to achieve better learning results. In summary, due to the immersion, interactivity, and visualization of virtual reality technology, comparing students with ordinary English learning can effectively stimulate students' interest in learning, thereby improving students' learning enthusiasm, and can be effective even if feedback is timely. Students are given appropriate positive reinforcement and negative reinforcement (positive reinforcement), which can ultimately greatly improve students' English learning ability.

With the popularization of mobile terminal equipment and the rapid development of mobile communication technology, computing-intensive services have placed high requirements on the computing and storage capabilities of the terminal. Therefore, many researchers consider introducing the idea of cloud computing into mobile communication networks, using mobile cloud computing to 
accelerate the operation and processing of computing-intensive services to improve user experience. By adopting English teaching courses, students' interest in the classroom can be improved. Using the interactive nature of virtual software can also give full play to students' subjective initiative. In addition, it also meets the needs of school education equipment update.

Zhao C used a real human-computer interaction scheme, used unity $3 \mathrm{D}$ game engine to build virtual reality scenes, defined shaders to improve scene rendering effects, and used Oculus Rift DK2 to complete an immersive 3D scene demonstration [1]. Sherazi H H clarified the feasibility of a completely cable-free IoT deployment. However, in order to save costs, the practicability of the configuration will become worse, leading to safety problems [2]. Zhang strengthened his experiments and conducted effective explorations on the basis of cooperation with schools and enterprises [3].

The innovation of this article lies in the application of virtual reality technology in English teaching and other areas where people have less researched. Four different research methods are used to conduct research to ensure the accuracy of the entire research results.

\section{English Teaching Methods}

\subsection{Methods of English Teaching Research}

2.1.1. Investigation and Research Methods. The appropriateness of the design and how to implement the design are explained. At the beginning of the course, the effectiveness of developing virtual experiments in the English teaching system was mainly analyzed through research. During the course, we will continue to consult frontline teachers [4].

2.1.2. Field Research Method. In-depth multimedia learning in experimental courses mainly introduces the experiment teaching of the multimedia system, learns the teaching method of the teacher in the experiment class, analyzes the operation method of the students in the experiment process, understands the learning difficulties of the students, and determines the focus of the graduation design [5].

2.1.3. Action Research Methods. Starting from this teaching and training practice, plan and implement its functions in detail. After obtaining the raw materials, the system will be gradually designed and produced. The original idea will always have new problems in practice. For these issues, we will continue to consult the literature and inform teachers and classmates. Consult, explore, and solve one by one in the production process [6].

2.1.4. Evaluation and Investigation Method. The achievement of the planning objectives of this study is analyzed and evaluated. And the feedback data of students are collected through questionnaire surveys and interviews and used on the basis of statistical analysis. [7].
These four survey methods are different. Field investigation is also called field investigation or field research, which is a concept in the field of communication. The investigation and research method refers to the direct acquisition of relevant materials through investigation and understanding of objective conditions, without being restricted by time and space. The action research method combines pure educational scientific research experiments with quasi-educational scientific research experiments, relying on its own educational and teaching practice. The investigation and evaluation method is also called the "written investigation method" or the "form-filling method." An investigation method collects research materials indirectly in written form. The results of this method are more affected by the factors of the respondents than the above three methods. This article mainly uses the survey research method and the survey evaluation method to be more in line with the actual situation.

\subsection{Advantages of Using VR Technology in English Teaching.} When considering the communication scheme between the cloud computing center and edge devices, in order to enable the bottom-level data to be accurately and timely transmitted to the upper-level center, and for the timely transmission of upper-level commands to the edge devices, a communication system with a strong network signal is required. Among them, one or more standard network communication interfaces have become a necessary requirement for the system. Therefore, TCP/IP protocol support is needed. This is because only TCP/IP allows the device to fully connect to the Internet. Socket is a commonly used API technology in TCP/IP network, which allows the computer to communicate with other devices with Socket interface through this port. Therefore, after comprehensively considering the various requirements mentioned above, the system chooses to use the Socket communication method under the TCP/IP network to establish the communication link between the edge device and the cloud computing center. Due to the limitations of English teaching, some teaching activities may not be carried out [8]. An immersive environment can significantly improve the efficiency of language learning because such an environment requires learners to continuously receive and output new languages, and VR allows you to experience foreign language communication even in a Chinese classroom environment. Unimersiv is one of the representatives of this application. This software can be used with Oculus Rift. Users can communicate with users all over the world in their own rooms and improve their foreign language skills in a real foreign language environment. VR technology can simulate a virtual environment through a computer or the like. Teachers and students of the college can use virtual reality equipment to enter the virtual teaching environment.

Introduction of VR technology into teaching can change traditional teaching methods. Virtual reality equipment teaching is more intuitive and specific, enriching the teaching content. According to recent news reports, a group of researchers from the University of California, Davis and 
Los Angeles have invented a virtual reality technology that can be used in the education field to display topographic sandboxes in 3D. And this kind of sand table based on "Augmented Reality" technology even allows visitors to move the hills with their hands. It can deconstruct technical action teaching, solve the difficulties of traditional teaching, and improve teaching effects.

VR technology can measure the learning status of students and strengthen supervision. Applying virtual reality technology to college physical education can effectively promote digital teaching. When a student uses a VR device for learning, the VR device can monitor the student's learning in real time and upload the student's learning data to effectively measure the student's learning status. University professors can analyze student learning data, formulate targeted courses, and improve teaching effects [9].

\subsection{VR Technology 3D Collision Detection Acceleration} Algorithm. Collision detection is not only used but also has high application value in computer animation and robot control. The use of VR technology and 3D collision detection acceleration algorithms can make the images presented by virtual reality more lively and authentic. The collision detection problem can be divided into two-dimensional level detection and three-dimensional collision interval detection according to different positions, in which the moving object has been located. The 3D collision detection algorithm is more complicated, but the $2 \mathrm{D}$ level detection can often provide specific basis and inspiration for the 3D collision detection. The research field of collision problem includes two aspects: conflict response and conflict detection. The content of conflict response investigation belongs to the field of mechanical research [10]. The research on conflicts in three-dimensional space generally has two aspects: collision and collision avoidance. The so-called collision problem refers to whether many objects collide when moving along a given path in space. Avoidance of conflict refers to the movement of two or more objects without colliding. The application of the collision detection algorithm to English teaching is embodied in three-dimensional collision, which provides students with a virtual space, which is conducive to creating a better atmosphere in English teaching mode.

\subsubsection{Collision Detection Algorithm Based on Fixed Time} Slice Length. This algorithm divides the target moving process into multiple time periods, each of which has the same length, and performs collision detection at the end of each time. The key here is the choice of time interval. If the length of the time interval is too long, there is a possibility of exposure, there is a conflict in the time interval, but it is not detected [11]. However, if the time span is too short, conflict detection will be very frequent. The time limit is as follows:

$$
\frac{\min \left(b_{i}\right)+\min \left(d_{i j}\right)}{\max \left(v_{i}\right)} \geq \Delta t(i, j=1,2, \ldots, n) i \neq j .
$$

In order to effectively solve the high network load, high bandwidth, low latency, and other requirements brought about by the rapid development of mobile Internet and Internet of Things, the concept of mobile edge computing (MEC) was proposed, and it has received extensive attention from academia and industry. Among them, the number of objects in the virtual space is represented by $n$, $b_{i}$ is the volume of the $\mathrm{i}$-th object, the distance between two objects in the space is represented by $d_{i j}$, and $v_{i}$ is the maximum speed of the first object. $\Delta t$ represents the length of the time slice.

The bounding box method is currently a commonly used collision detection method. It uses logical space boundary frames to wrap virtual objects. Since the bounding box has a simple shape, generally rectangular and parallel, it is easy to judge whether there is a spatial overlap between them. Its biggest advantage is that it can quickly detect conflicts. It is very effective to prove that two objects do not intersect. However, if a conflict between the boundaries is detected, it cannot be determined whether there is a conflict between the objects. We call this a situation where a conflict is detected through improper calculations, but in fact no conflict is displayed as a false alarm. Because the bounding box is only a rough representation of the boundary of the object, we can call this detection "rough detection" [12]. Generally speaking, reducing the number of false alarms is an important criterion for selecting and creating boundary boxes. In some cases where high-precision interactive functions are required, it is necessary to further identify and calculate the positional relationship between objects, which is to perform accurate collision detection. However, it is clear that better tracking algorithms can significantly reduce the overall cost of the system.

\subsubsection{Collision Detection Algorithm with Variable Time Slice} Length. When the space collision frequency is low, this method can save a lot of calculation time. The idea of this method is that if the minimum distance and speed limit between two objects are known, the lower limit of the collision time can be calculated; that is, the collision cannot occur before [13]. At this point, the virtual time of the system can be performed immediately without any collision detection. When two objects are far apart, the time period may be larger; when two objects are closer, the time period must be smaller. The silent condition here is that relative to the distance between the objects, the space volume of the object is small, so the space volume of the object can be ignored.

\subsubsection{Object Collision Detection Algorithm for "Scanning} Entities". These objects represent the volume of space occupied by objects when they move on a given orbit. This algorithm transforms space-time problems into space problems. When the collision probability is a small probability event, it can effectively reduce the number of calculations and the complexity of each calculation, but more false alarms are because the new entity is intersecting, which does not mean that the two objects arrive at the intersection at the same time. Under the current computer hardware conditions, the fixed time interval collision detection 
method is more suitable for real-time display of a graphics computer. The fixed time method should be more flexible in determining the impact time. In software applications, the conflict detection algorithm using entity scanning requires a separate additional step to create the scanned entity, which requires a certain computational overhead [14].

2.4. Mobile Edge Computing. Mobile edge computing can provide IT and cloud computing functions in the wireless access network near mobile users. For application developers and content providers, MEC can provide a very lowlatency and high-bandwidth service environment at the edge of the wireless access network and can directly access the real-time information of the wireless network (such as user location and cell load). It can be seen that mobile edge computing can speed up the distribution of content and improve the response speed of services and applications by providing auxiliary computing resources, thereby enhancing and enriching the user experience. Operators can also open the MEC server deployed at the edge of the wireless network to third-party partners, so that they have the ability to quickly deploy innovative applications and services to mobile users, enterprises, and other vertical fields. The proximity of the MEC server to users, the flexibility of deployment, and the powerful computing power much higher than that of mobile terminals enable mobile operators, service, and content providers, OTT (OverTheTop, OTT) players, and independent software vendors. Internally, they create greater profit opportunities through the complementarity of their respective business models.

Mobile edge computing and collision detection algorithms are important methods to make and detect whether the images presented by virtual reality are real. Although these two methods have played a very good role, they also have disadvantages such as difficulty in use.

\section{VR Technology English Teaching Experiment}

\subsection{Virtual Experiment Framework of English Teaching} System. Converting the original C/S structure to a threelayer $\mathrm{B} / \mathrm{S}$ structure greatly reduces the pressure on customers. In this architecture, the user interface is implemented through the 2345 browser program [15]. Few transaction logics are applied to the front end (browser), but the main logic of the transaction is applied from the server side (server). The $\mathrm{B} / \mathrm{S}$ architecture method is a fine customer operation, which simplifies the customer software. The client only needs basic application configuration and loading specific additives to be the client application operating platform. The increase in the client browser program can be easily obtained through the network, and the operation of the software has nothing to do with the system platform [16]. The frame diagram is shown in Figure 1.

The English teaching virtual experiment system is developed under the current campus network environment. University users can quickly connect to the system through the campus network for operation, and external users can

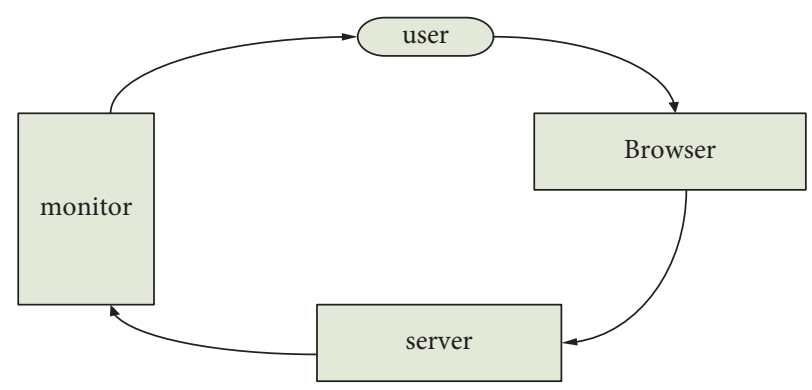

FIgURE 1: The overall framework of the virtual system.

also have access to the campus network application server through the Internet to connect to the system for learning. The maintenance of the virtual experimental system can only be realized by operating the server, such as improving the experimental operation process, increasing the experimental function unit, and expanding the experimental function [17].

The main code of this virtual experiment system is a VRML file. As the second-generation network application language, VRML should be transmitted in binary format. Transferring VRML files to the B/S architecture system does not require high network requirements. In the current network environment, it can fully respond to the fast transmission of code files [18]. In addition, the code files are executed on the client in an explanatory manner, which requires extremely low computer configuration for the client. Once the client browser program installs a specific plug-in, the VRML file can enter the wonderful world of virtual reality for execution.

(1) Data Collection Function. The data collection module is the source of data in various data mining systems.

(2) Data Preprocessing Function. The original data collected by the data acquisition module include not only the data required for subsequent processing but also various redundant data and incidental noise signals. For redundant and noisy data, we have carried out an average value solution, which will not have much impact on the experimental results of the article.

3.2. VR System Design Experiment. The virtual reality system based on the PC platform is mainly composed of a highperformance CPU, a tracker, a helmet-mounted display, a three-dimensional graphics accelerator card, a three-dimensional sound card, and a game joystick [19].

The use of computers to realize virtual reality technology is mainly used to develop virtual reality desktop systems. Generally speaking, a virtual reality system constructed by a computer has its unique characteristics: the computer can achieve a strong sense of presence, the current popular DVI technology can obtain $3 \mathrm{D}$ in real time in a virtual environment, and the computer can support various materials in the human-machine interface. This is a widely used manmachine interface. In addition, in this virtual reality system, three-dimensional data can support DXF files. Using these 
capabilities, the development of computer-based virtual reality technology application systems will be easier. Thousands of computers in the world can be used for the promotion of virtual reality technology and domestic development, which has also become the main focus of future virtual reality technology development. Although the current more complex virtual reality application systems need to be built on the basis of high-performance graphics platforms, real-time applications are not required for art and education, and the virtual reality of low-level desktop computer systems can meet the implementation requirements [20].

\section{English Teaching Analysis under VR Technology}

4.1. Analysis of English Teaching Attitudes of VR Technology. This article investigates the attitudes of 200 students with the same level of English proficiency towards the application of virtual reality technology to English teaching. The results of the survey are shown in Table 1.

Draw Table 1 into a more intuitive graph, as shown in Figure 2. Through the analysis of Figure 2, it can be concluded that 116 students are very satisfied with VR technology-assisted English teaching, accounting for $58 \%$ of the total. VR technology can enhance their interest in learning, thereby improving the efficiency of learning [21]. Among them, 56 students were generally satisfied with VR technology-assisted English teaching, accounting for $28 \%$ of the total, while 18 students were dissatisfied with the VR technology-assisted English teaching form, accounting for $9 \%$ of the total. In addition, there are also 10 people who are very dissatisfied with VR technology-assisted English teaching, accounting for $5 \%$ of the total $[22,23]$.

Based on the above data, more than half of students and teachers believe that multiple teaching methods are necessary. About $66.7 \%$ of teachers think it is necessary, and about $57.7 \%$ of students think it is necessary [24]. Through analysis, it can be found that teachers pay more attention to multiple technologies and understand their advantages in English teaching. As for whether VR technology is good or not, it depends on how teachers use VR technology [25].

Although the average scores of students have been rising, the increase is not very large. Under the assisted English teaching mode, students' average English scores have been significantly improved, which also reflects the practicality of VR technology, which can quickly and effectively improve students' performance and adaptability to English problems. The average score of students in English has increased from 63 to 92 , which is a suitable way for students to teach English [26]. The specific comparison of the three teaching modes is shown in Figure 3.

4.2. Analysis of the Impact of VR Technology on English Teaching. Calling the cloud computing center to complete big data operations and timely feedback to the edge devices is the biggest advantage of edge computing. It includes four aspects: English understanding, resource sharing, lively and interesting, and the right to choose. Similarly, the influence of traditional methods on English teaching is also divided into these four aspects. Therefore, an analysis and comparison of the degree of influence of the two on English teaching were made. The results are shown in Figure 4.

According to the data analysis in the figure, it can be concluded that the greatest impact of VR on English teaching is vivid and interesting, accounting for 78\%. This also shows that VR can allow students to immerse themselves in their own world and find their own fun. The right to choose is the least impact of VR on English teaching, accounting for only $21 \%$. After all, the teaching mode is still in the hands of teachers. What VR can do is to provide more choices [27]. Comparing traditional English teaching and VR English teaching, it can be seen that the impact of VR on English teaching is higher than traditional English teaching in all aspects. We tested the students' English learning ability through the test question test method and used the average algorithm to get the average score of the student, so as to compare the changes of the students' learning ability before and after learning. English comprehension ability is 19\% higher, and the degree of resource sharing is $11 \%$ higher. The degree of vividness and fun is $20 \%$ higher, and the right to choose is $9 \%$ higher.

Virtual reality supports multiple people online at the same time. Students can communicate with each other, cooperate with each other, discuss certain issues, and share learning resources and experiences in a virtual environment. And this technology can make students more interested in learning English. The humanized learning plan activities have largely broken the traditional teaching's restrictions on students' thinking and communication, allowing it to implement multiple communication that uses auditory, speech, and sensory systems for learning. Students are encouraged to understand language learning problems and solve them based on their own social experience. The students deeply felt the cultural charm of English. Mastering the basics is very enlightening. Students have a lot of autonomy in the virtual environment. By manipulating these factors, they can communicate with celebrities from different countries at any time, and they can also communicate with their idols. This interesting learning method is more suitable for students and more effective for language learning.

4.3. Analysis of VR Technology English Teaching System. In this test, 150 questionnaires were issued, 120 questionnaires were returned, and the recovery rate was $80 \%$. Among the returned test questionnaires, 20 were invalid questionnaires and 100 valid questionnaires, with an effective rate of $50 \%$. This questionnaire is a survey and analysis of the English teaching system. The results are shown in Table 2.

In order to analyze the data more intuitively, draw the table into a graph as shown in Figure 5.

By analyzing the data in the figure, we can get the following:

(1) The popularization of VR technology is relatively narrow, and students know very little about the experimental teaching and development of the use of 
Table 1: Attitudes of VR technology applied to English teaching.

\begin{tabular}{lcccc}
\hline Evaluation & Very satisfied & Generally satisfied & Not satisfied & Very dissatisfied \\
\hline Number of people & 116 & 56 & 18 & 10 \\
Percentage & $58 \%$ & $28 \%$ & $9 \%$ & $5 \%$ \\
\hline
\end{tabular}

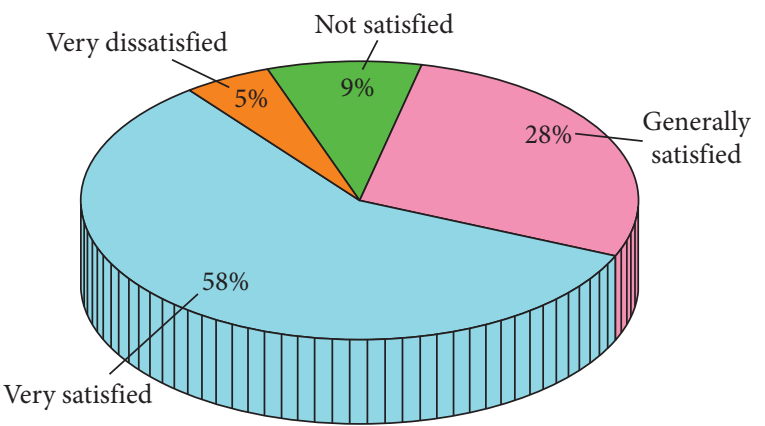

FIgURE 2: Evaluation of VR technology applied to English teaching.

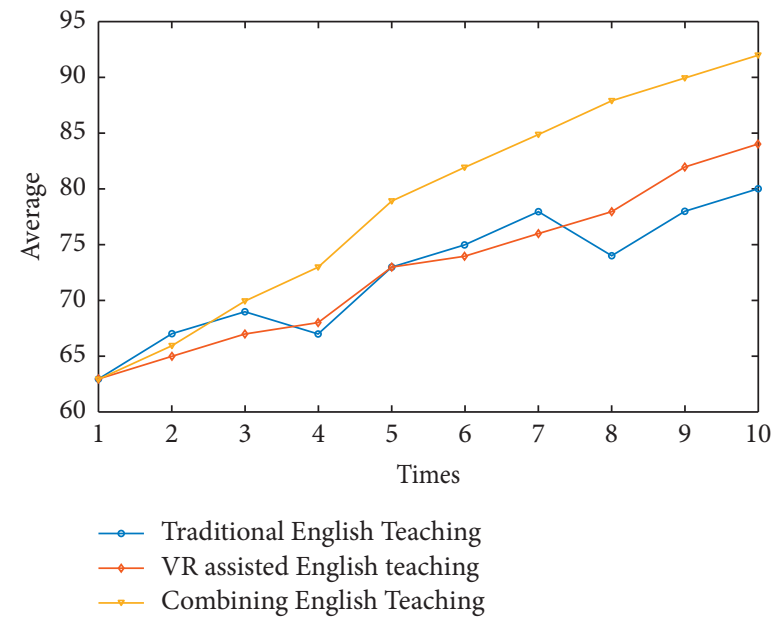

FIgURE 3: Comparison of the effects of three English teaching modes.

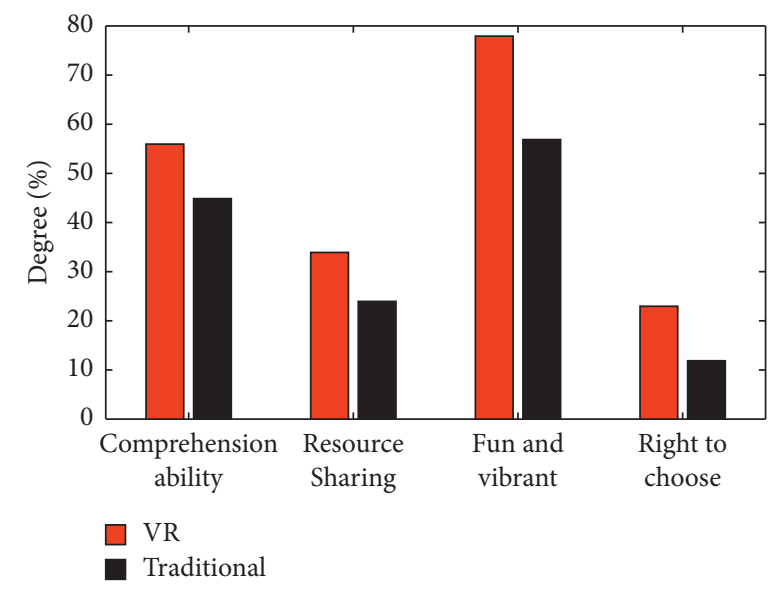

Figure 4: The impact of VR technology on English teaching. 
TABle 2: Analysis results of the VR technology English teaching system.

\begin{tabular}{lccc}
\hline Problem index & & Result percentage (\%) & \\
VR technology & Clear (2) & Know a little (37) & Do not know (61) \\
\hline Mastery of equipment & Fully capable (42) & Need to be strengthened (39) & Cannot (19) \\
Virtual reality & It is good (49) & General (30) & Difference (21) \\
Virtual experiment operation & Done (0) & Have seen it before (14) & Never seen it (86) \\
\hline
\end{tabular}

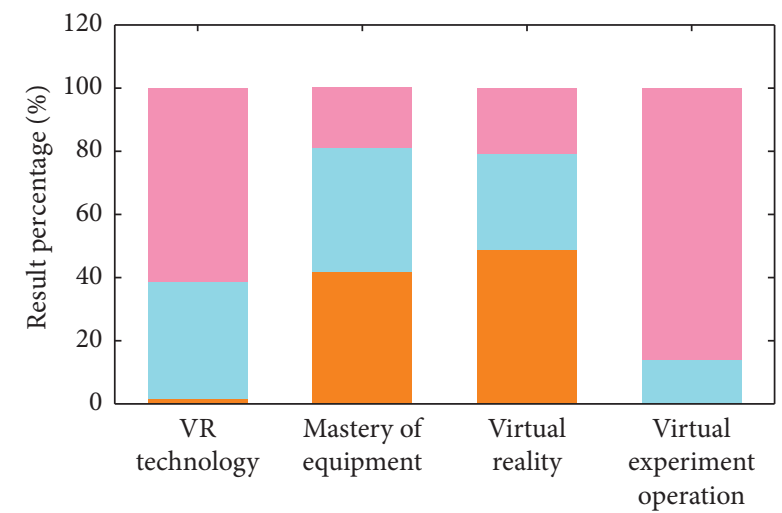

FIGURE 5: VR technology English teaching system analysis results.

VR technology, and only $2 \%$ of them are clear about VR technology mainly because VR technology has only developed rapidly in recent years. A computer technology is rarely used in real life, which shows that it has great potential for development.

(2) It can be seen from the results that $81 \%$ of the students have a certain understanding and knowledge of these English teaching system equipment after conducting the virtual experiment operation. They get started quickly when they enter the laboratory for experimental operations after practice. Many students do not need to listen to the teacher's explanation but can carry out independent experimental operations and also have an understanding of the matters that should be paid attention to in the experiment, such as the correct operation of the projector on and off and the range of the moving parts of the video showcase. However, due to the fact that some students have less contact with computers, these students account for $19 \%$ of the total. This is reflected in the trials of some students in the School of Music and Sports. During the operation, the purpose was not clear during the learning process, so after the virtual experiment, I was not impressed by the specific experimental phenomenon corresponding to the device function buttons, and many problems occurred in the actual experimental operation.

(3) In the course of the questionnaire, the author also interviewed some students from these colleges. Most of them had never touched the English teaching system equipment before taking this course, and they knew little about the valuable equipment such as video display stands and projectors.

\section{Conclusions}

Starting from the current status of English system experimental teaching, this paper uses modern media theory as the guidance of behavior and cognitive learning theory, combined with the development process of this virtual experimental system, and discusses the importance of virtual reality technology in experimental teaching. On the basis of a comprehensive understanding of mainstream virtual reality software technology, combined with the development of virtual experimental systems, some research studies have been carried out and, on this basis, a virtual experimental platform of multimedia teaching system has been completed, allowing students to conduct experimental research and master experimental skills. The interactive functions of virtual devices and the construction of virtual scenes have been studied and discussed in depth. Based on a variety of application technologies, a virtual experimental operation platform with a certain sense of immersion, interaction, and imagination is constructed in a more reasonable way.

For this reason, edge data processing technology with edge computing as the core was produced and widely promoted. Edge computing is defined as a distributed open platform that integrates core capabilities of network, computing, storage, and application on the edge of the network close to the source of things or data. In fact, edge computing is a new ecological model. By converging five types of resources such as network, computing, storage, application, and intelligence at the edge of the network, it can improve network service performance and open network control capabilities, thereby inspiring something similar to the mobile Internet. Many theoretical and practical issues are still under study. This article has made some useful attempts on the application of virtual reality technology in virtual experiment teaching, but it needs further improvement.

This article mainly studies the application of VR technology in English teaching. After studying the types of VR technology characteristics and actual teaching cases, the concept and teaching purpose of virtual reality vehicle intervention technology in the experimental modeling course are proposed, and its construction content and teaching methods are explained. The technical means of desktop virtual reality teaching are summarized, and finally desktop virtual reality tools are constructed to create virtual reality desktops for desktop computers and allow them to appear in the virtual reality of the classroom. Future work will focus on caching data on the edge server, but due to the limited memory of the storage server at the edge, the hit rate of the edge cache is limited. Therefore, edge processing services can 
be added to the edge to further improve service performance, providing theoretical support and motivation for the research of wireless video transmission systems based on edge computing.

\section{Data Availability}

This article does not cover data research. No data were used to support this study.

\section{Conflicts of Interest}

The author declares that there are no conflicts of interest.

\section{Acknowledgments}

This work was supported by Hunan Education Department, Key Research Projects of Hunan Education Department: Research on the Cultivation Path of Excellent English Normal University Students in Local Universities under the Times of New Liberal Arts No. 19A462 and Teaching Reform Research Project of General University: the "four in one" Practical Research on the Cultivation of Teaching Ability of English Normal University Students in the Information Age No. 894. This work was also supported by Chenzhou Government: Key Research and Technological Innovation Projects of Science and Technology Bureau: the Reform of Rural Education in Chenzhou from the Perspective of Big Data No. ZDYF2020157. Key research Project of Education Bureau: On the Joint Training of Excellent English Teachers in Chenzhou by Universities, Local Governments, and Middle Schools No. CJK20XXHZ01. The Project of Social Science Office: the Training Mechanism and Path of Primary English Teachers in Chenzhou No. Czssk12019025.

\section{References}

[1] C. Zhao, "Application of virtual reality and artificial intelligence technology in fitness clubs," Mathematical Problems in Engineering, vol. 2021, no. 20, pp. 1-11, 2021.

[2] H. H. R. Sherazi, G. Piro, L. A. Grieco, and G. Boggia, "When renewable energy meets LoRa: a feasibility analysis on cableless deployments," IEEE Internet of Things Journal, vol. 5, no. 6, pp. 5097-5108, 2018.

[3] Y. Zhang, "Ideas and approaches on "construction of high level simulation experimental teaching center of virtual chemical laboratory," IOP Conference Series: Earth and Environmental Science, vol. 94, no. 1, Article ID 012070, 2017.

[4] H. Zhang, Y. Zhang, Y. Gu, D. Niyato, and Z. Han, "A hierarchical game framework for resource management in fog computing," IEEE Communications Magazine, vol. 55, no. 8, pp. 52-57, 2017.

[5] D. C. Klonoff, "Fog computing and edge computing architectures for processing data from diabetes devices connected to the medical Internet of things," Journal of Diabetes Science and Technology, vol. 11, no. 4, pp. 647-652, 2017.

[6] V. R. Dushin, V. F. Nikitin, N. N. Smirnov, E. I. Skryleva, and V. V. Tyurenkova, "Microgravity investigation of capillary driven imbibition," Microgravity Science and Technology, vol. 30, no. 4, pp. 393-398, 2018.
[7] S. J. Basha and V. R. Kumar, "Design of MWCNT based through silicon vias with polymer liners to reduce the crosstalk effects," ECS Journal of Solid State Science and Technology, vol. 9, no. 4, Article ID 041002, 2020.

[8] G. S. P. K. Reddy, V. R. Rao, and A. V. Rao, "Effect of bracings in controlling the structural response under seismic force," International Journal of Civil Engineering and Technology, vol. 9, no. 7, pp. 170-181, 2018.

[9] T. B. Chowdary, D. K. Babu, and V. R. Rao, "Damage assesement curves for $\mathrm{rc}$ framed structures under seismic loads," International Journal of Civil Engineering and Technology, vol. 9, no. 8, pp. 1769-1782, 2018.

[10] V. R. Anderson, N. Nepal, S. D. Johnson et al., "Plasmaassisted atomic layer epitaxial growth of aluminum nitride studied with real time grazing angle small angle $\mathrm{x}$-ray scattering," Journal of Vacuum Science \& Technology A: Vacuum, Surfaces, and Films, vol. 35, no. 3, Article ID 031508, 2017.

[11] N. Alias, J. Mai, and H. Musa, "Nanotechnology theory used for simulation of emerging big data systems on high performance computing: a conceptual framework," Journal of Theoretical and Applied Information Technology, vol. 95, no. 22, pp. 6147-6162, 2017.

[12] T. Q. Dinh, J. Tang, and Q. D. La, “Offloading in mobile edge computing: task allocation and computational frequency scaling," IEEE Transactions on Communications, vol. 65, no. 8, pp. 3571-3584, 2017.

[13] P. L. Suryawanshi and V. R. Pawar, "Design of low power pierce crystal oscillator using CMOS technology," International Journal of Computer Sciences and Engineering, vol. 6, no. 6, pp. 421-423, 2018.

[14] G. R. Sekhar and S. Chakravorty, "TESL/TEFL: teaching English as a second or foreign language[J]," English Journal, vol. 54, no. 5, pp. 414-418, 2017.

[15] V. A. Rozhina and T. A. Baklashova, "Teaching English language to young school-age children while making projects, playing games and using robotics," XLinguae, vol. 11, no. 1, pp. 102-113, 2018.

[16] V. R. Ravi and S. Aathithya, "Speed control of solar powered separately excited DC motor[J]," International Journal of Computing \& Information Technology, vol. 11, no. 1, pp. 1323, 2019.

[17] A. Burak and Y. Y. Tu Ba, "The effect of flipped classroom model on students' classroom engagement in teaching English," International Journal of Instruction, vol. 11, no. 2, pp. 385-398, 2018.

[18] Y. Zhou, K. Su, and L. Shao, "Reform and exploration of virtual experiment teaching in the course of modern control system," Agro Food Industry Hi Tech, vol. 28, no. 1, pp. 1904-1908, 2017.

[19] V. R. Fanaie, M. Karrabi, and M. M. Amin, "Biosorption of 4chlorophenol by dried anaerobic digested sludge: artificial neural network modeling, equilibrium isotherm, and kinetic study," International Journal of Environmental Ence \& Technology, vol. 14, no. 1, pp. 1-12, 2017.

[20] J. Chen, J. Wang, J. Zou, H. Lv, X. Hu, and Y. Xu, "Polycapillary coupled X-ray digital radiation imaging system: feasibility analysis," Nuclear Instruments and Methods in Physics Research Section A: Accelerators, Spectrometers, Detectors and Associated Equipment, vol. 870, no. 21, pp. 19-24, 2017.

[21] X. He, Y. Chu, A. Lindbråthen, M. Hillestad, and M.-B. Hägg, "Carbon molecular sieve membranes for biogas upgrading: 
techno-economic feasibility analysis," Journal of Cleaner Production, vol. 194, no. 1, pp. 584-593, 2018.

[22] Z. Lv, D. Chen, R. Lou, and Q. Wang, "Intelligent edge computing based on machine learning for smart city," Future Generation Computer Systems, vol. 115, pp. 90-99, 2021.

[23] S. Wan, L. Qi, X. Xu, C. Tong, and Z. Gu, "Deep learning models for real-time human activity recognition with smartphones," Mobile Networks and Applications, vol. 34, pp. 1-13, 2019.

[24] Y. Yihdego and R. A. A. Weshah, "Treatment of world's largest and extensively hydrocarbon polluted environment: experimental approach and feasibility analysis," International Journal of Hydrology Science and Technology, vol. 8, no. 2, pp. 190-208, 2018.

[25] L. Kumar, R. K. Behera, S. Rath, and A. Sureka, "Transfer learning for cross-project change-proneness prediction in object-oriented software systems," ACM SIGSOFT Software Engineering Notes, vol. 42, no. 3, pp. 1-11, 2017.

[26] B. Wang, X. Yang, and G. Zhang, "Key technologies of DP float-over installation and corresponding feasibility analysis in the east China sea," Ship Building of China, vol. 58, no. 1, pp. 162-169, 2017.

[27] W. Fengxu, "Computer distance virtual experiment teaching application based on virtual reality technology," International Journal of Emerging Technologies in Learning (iJET), vol. 13, no. 4 , p. 83, 2018. 